\title{
Primary Cutaneous Mucinous Carcinoma of the Vulva Displaying a Papillary Pattern
}

\section{Aditi Ranade* and Antonio Macias}

MD, Department of Pathology, St. Luke's - Roosevelt Hospital Center, Columbia University College of Physicians \& Surgeons, New York, USA

\begin{abstract}
Primary cutaneous mucinous carcinoma (PCMC) is an extremely rare, low grade, adnexal sweat gland neoplasm that commonly affects the head and neck in the elderly population. Microscopically, it typically shows islands of epithelial cells floating in pools of mucin separated by fibrous septae. Recurrence after excision is common but metastasis is rare. Cutaneous mucinous carcinoma of the vulva is an extremely rare tumor. We report here one such case in an 89 year-old female which on histology revealed a multicystic tumor with tumor cells arranged in the form of papillae, floating in pools of mucin within the cysts. Our case thus illustrates an uncommon histological pattern of vulvar PCMC and discusses its clinical significance.
\end{abstract}

Keywords: Cutaneous mucinous carcinoma; Papillary pattern; Metastatic adenocarcinoma; Bartholin's gland carcinoma; Ectopic breast tissue in the vulva

\section{Introduction}

Primary mucinous carcinoma of the skin is an uncommon but histologically distinct adnexal neoplasm that mainly affects the head and neck in the elderly population [1,2]. Primary cutaneous mucinous carcinoma of the vulva is extremely rare and very rarely shows a papillary pattern on histology. This, along with the rare location in the vulvar skin brings into consideration multiple possibilities of origin.

\section{Case Report}

Our patient an 89-year-old G5 P4, presented to the Gynecology Department of our hospital with a several month history of gradually increasing vulvar swelling on the left labium majus. The initial clinical impression was that of an epidermoid cyst (Figure 1). There was no family history of breast or gynecological malignancy. Her past surgical history was significant for a total abdominal hysterectomy and bilateral salpingo-oophorectomy for prolapse (in 1975) and right breast lumpectomy (1995) followed by a mastectomy (2000) for an intraductal carcinoma. Follow-up mammograms in 2005 and 2007 were unremarkable. Her physical examination, laboratory evaluation and imaging studies (Computerized Tomographic (CT) scan of the thorax, abdomen and pelvis) including a colonoscopy were essentially unremarkable.

The left vulvectomy specimen consisted of a $2.8 \times 2.5 \mathrm{~cm}$ gray tan multicystic tumor with a gelatinous cut surface. There were no other skin lesions or lymphadenopathy and the labia minora and interlabial sulcus were unremarkable. Histological examination of the vulvar lesion showed a multicystic tumor involving the dermis and subcutaneous tissue, with tumor cells arranged in the form of papillae floating in pools of mucin within the cysts. The papillae contained fibrovascular cores (Figure 2-b). Also seen were thin fibrous septae separating the papillary fragments. Occasional tight cell clusters were also seen. Cytologically, the cells showed mild pleomorphism with oval to elongated nuclei, hyperchromasia and occasional prominent nucleoli (Figure 2-c). No perineural or lymphatic permeation was identified and the surgical margins all around were clear on pathological examination.

On immunohistochemistry, the tumour cells were reactive for cytokeratin-7 (CK-7), epithelial membrane antigen (EMA), carcinoembryonic antigen (CEA) and estrogen receptor protein
(ER) (Figure 2 - d, e and f). MUC1 immunostain was positive in the apical mucin secreting portion of the tumor cells. The negative stains were, cytokeratin -20 (CK-20), chromogranin and synaptophysin, progesterone receptor protein (PR), CA 19-9, CK-5/6 and D2-40. The mucin within the cysts stained with mucicarmine, colloidal iron and Alcian blue at a $\mathrm{Ph}$ of 2.5 (Figure 2- $\mathrm{g}$ and i). It was PAS positive and diastase resistant (Figure 2- h). It was thus a non-sulfated type of mucin. This immunohistochemistry, coupled with the negative results of an extensive clinical examination and imaging studies, helped us rule other mucinous lesions and metastatic mucinous carcinoma of the vulva. Hence, a diagnosis of primary cutaneous mucinous carcinoma of the vulva was made. On follow up examination, the patient was free from recurrence of her disease and did not require any additional therapy.

\section{Discussion}

Primary cutaneous mucinous carcinoma (PCMC) typically presents as a slowly growing nodule in the head and neck region in the elderly individuals [1,2]. A vulvar location of this tumor is extremely rare and has been documented only in rare case reports [3]. Microscopically the tumor shows cell clusters most often arranged in the form of trabeculae, cords, cribriforming nests, all floating in pools of mucin separated by thin fibrous strands [4,5]. Our case however showed a multicystic tumor, with cells arranged in the form of papillary fragments having fibrovascular cores and floating in pools of mucin contained within the cysts. To the best of our knowledge, this kind of a papillary architecture is extremely rare in vulvar cutaneous mucinous carcinomas.

Most cases of mucinous carcinoma in the skin are secondary. Mucinous carcinoma metastatic to the skin may arise from the breast, gastrointestinal tract, pancreas, lung, salivary gland and

*Corresponding author: Aditi Ranade M.D, Department of Pathology, Clark 4 New York, NY 10025, USA, Tel: 5134107111; Fax: (212)5235678; E-mail: aranade@chpnet.org, aditiranade@hotmail.com

Received March 25, 2011; Accepted April 10, 2011; Published April 13, 2011

Citation: Ranade A, Macias A (2011) Primary Cutaneous Mucinous Carcinoma of the Vulva Displaying a Papillary Pattern. J Cytol Histol 2:113. doi:10.4172/21577099.1000113

Copyright: (C) 2011 Ranade A, et al. This is an open-access article distributed under the terms of the Creative Commons Attribution License, which permits unrestricted use, distribution, and reproduction in any medium, provided the original author and source are credited. 
Citation: Ranade A, Macias A (2011) Primary Cutaneous Mucinous Carcinoma of the Vulva Displaying a Papillary Pattern. J Cytol Histol 2:113. doi:10.4172/2157-7099.1000113

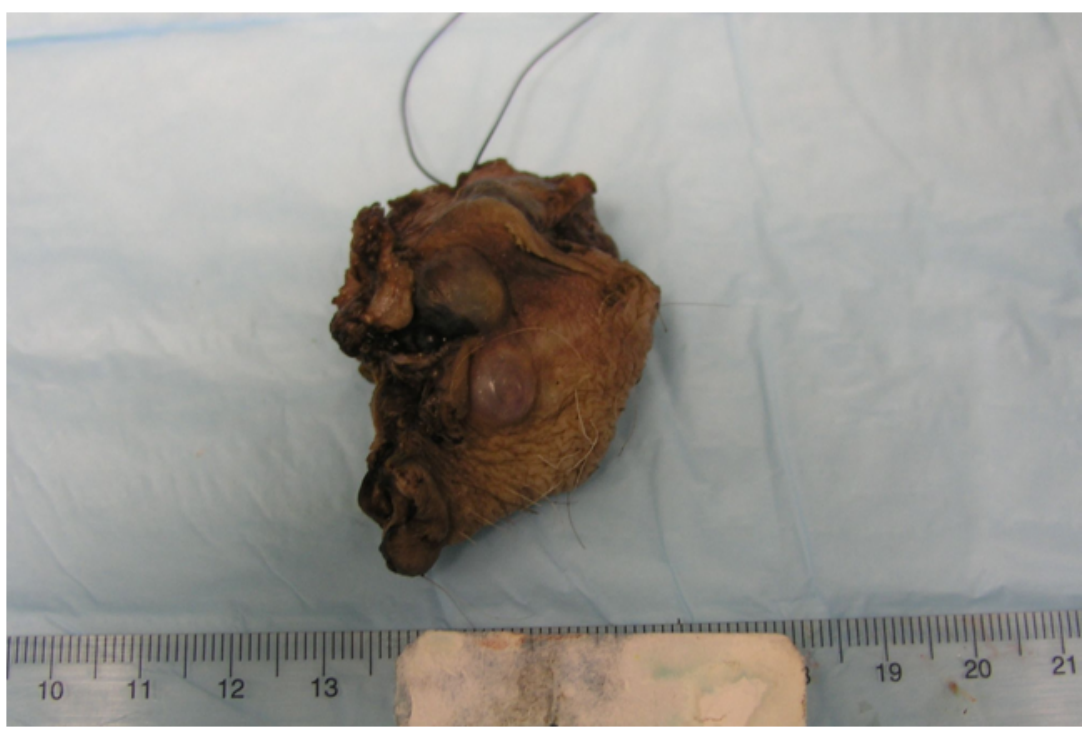

Figure 1: Gross image of the tumor.
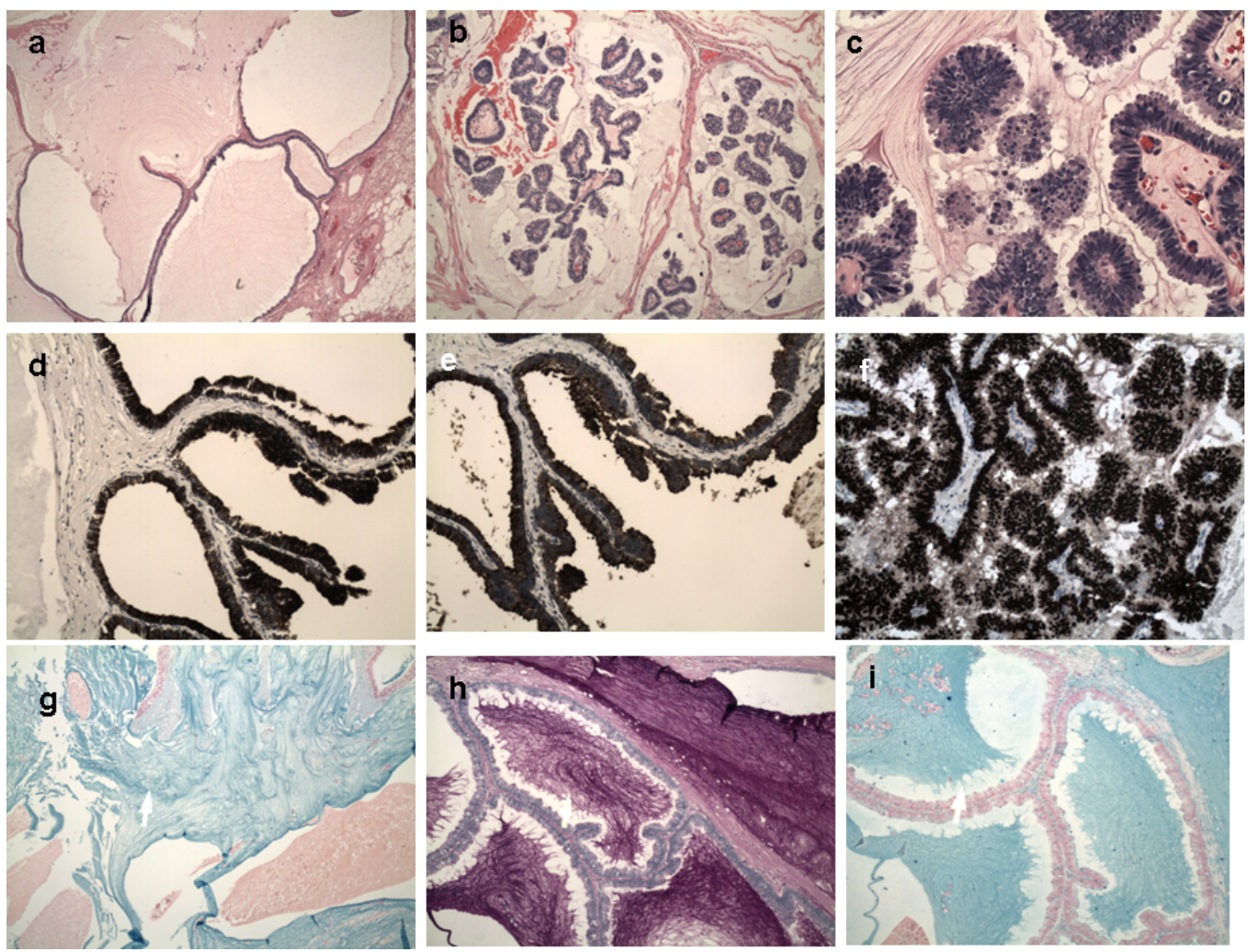

Figure 2: Microscopic images.

(a\&b) Cystic and papillary pattern, papillae with fibrovascular cores; c) Cells show elongated to oval nuclei with hyperchromasia and occasional prominent nucleoli; d) CK-7; e) EMA ; f) ER; g) Alcian blue at Ph 2.5; h) DPAS; i) colloidal iron.

ovary. Cutaneous metastasis from breast carcinoma are however very uncommon and metastasis from gastrointestinal tumors and other sites can be distinguished by immunohistochemistry and on clinical basis [6]. Therefore, before rendering a diagnosis of primary cutaneous mucinous carcinoma, a thorough search for mucinous carcinoma in other organs should be done, as was done in our case. Based on the histological pattern noted in our case, metastatic ovarian cancer was high on the differential, but the patient had undergone a total abdominal hysterectomy and bilateral salpingo-oophorectomy. Also immunohistochemistry supported a PCMC. On account of the vulvar location, other differentials like Bartholin's gland mucinous carcinoma and mucinous carcinoma arising in ectopic breast tissue also come into 
Citation: Ranade A, Macias A (2011) Primary Cutaneous Mucinous Carcinoma of the Vulva Displaying a Papillary Pattern. J Cytol Histol 2:113. doi:10.4172/2157-7099.1000113

Page 3 of 3

play [3]. These can however be excluded by clinical and pathological examination. We did consider a remote possibility of origin of the tumor in ectopic mammary tissue in the vulva, but despite extensive sampling, no breast parenchyma was identified in the tissue resected and the tumor was negative for CK-5/6 and BRST. Adenocarcinoma of Bartholin's gland may show mucinous areas however; these tumors are readily identified by the gynecologist due to their origin in the labia minora, which was unremarkable in our case.

The biological course of PCMC's is variable, and a wide local excision is adequate in most cases [3].

\section{Conclusion}

Primary cutaneous mucinous carcinoma of the vulva poses difficulties in diagnosis due to its location, bringing into consideration various differential diagnosis including a metastatic origin. Microscopically, in addition to the previously reported patterns of trabeculae, cords, cribriforming and nesting, the tumor may also display a true papillary architecture.

\section{References}

1. Paradela S, Castiñeiras I, Cuevas J, Almagro M, del Pozo J, et al. (2008) Mucinous carcinoma of the skin: evaluation of lymphatic invasion with D2-40. Am J Dermatopathol 30: 504-508.

2. Kelly BC, Koay J, Driscoll MS, Raimer SS, Colome-Grimmer MI (2008) Report of a case: primary mucinous carcinoma of the skin. Dermatol Online $\mathrm{J} 14: 4$.

3. Rahilly MA, Beattie GJ, Lessells AM (1995) Mucinous eccrine carcinoma of the vulva with neuroendocrine differentiation. Histopathology 27: 82-86.

4. Weedon D (2009) Weedon's Skin Pathology. Churchill Livingstone.

5. Martinez SR, Young SE (2005) Primary Mucinous Carcinoma of the Skin: A Review. The Int $\mathrm{J}$ of Oncology 2: 2.

6. LeBoit PE, Burg G, Weedon D, Sarasin A (2006) Pathology and Genetics of Skin Tumours (IARC WHO Classification of Tumours). IARC Press. 